\title{
Experimental demonstration of a structured material with extreme effective parameters at microwaves
}

\author{
Mário G. Silveirinha, ${ }^{1, a)}$ Carlos A. Fernandes, ${ }^{2}$ Jorge R. Costa, ${ }^{2,3}$ and Carla R. Medeiros ${ }^{2}$ \\ ${ }^{1}$ Department of Electrical Engineering-Instituto de Telecomunicações, University of Coimbra, \\ 3030 Coimbra, Portugal \\ ${ }^{2}$ Instituto Superior Técnico-Instituto de Telecomunicações, Technical University of Lisbon, 1049-001 Lisbon, \\ Portugal \\ ${ }^{3}$ Departamento de Ciências e Tecnologias da Informação, Instituto Superior de Ciências do Trabalho e da \\ Empresa, 1649-026 Lisboa, Portugal
}

(Received 10 September 2008; accepted 10 October 2008; published online 30 October 2008)

\begin{abstract}
Following our recent theoretical studies [M. G. Silveirinha and C. A. Fernandes, Phys. Rev. B 78, 033108 (2008)], it is experimentally verified that an array of crossed metallic wires may behave as a nonresonant material with extremely large index of refraction at microwaves and may enable the realization of ultrasubwavelength waveguides. (C) 2008 American Institute of Physics.
\end{abstract}

[DOI: $10.1063 / 1.3012373$ ]

The design of materials with unusual electromagnetic properties has received a lot of interest in recent years, mainly because of the great potentials of structures with simultaneously negative permittivity and permeability. ${ }^{1}$ However, materials with extreme parameters or extreme properties, such as near zero permittivity, extreme anisotropy, or very large permittivity, may also have interesting applications in many problems such as tunneling through narrow channels and bends, ${ }^{2}$ subwavelength imaging, ${ }^{3}$ realization of magnetic materials in the visible domain, and cloaking. ${ }^{5}$

In a recent work, ${ }^{6}$ we have reported that a composite material formed by crossed metallic wires may have an anomalously strong interaction with electromagnetic waves and may enable the realization of materials with extremely large positive index of refraction. The geometry of a grounded slab of the structured material is depicted in Fig. 1(a). It was theoretically predicted in Ref. 6 that such grounded slab may interact with a wave with electric field polarized along the $x$-direction as a material with very large positive permittivity. This result is rather surprising since systems formed by long metallic wires are typically thought as materials with negative permittivity. Here, we experimentally verify the results of Ref. 6 and demonstrate that the proposed material may enable the realization of ultrasubwavelength waveguides.

To this end, a prototype of the structured material was fabricated using a layer by layer design and printed circuit techniques [Fig. 1(b)]. In our planar design, the metallic wires are replaced by printed metallic strips. The material is formed by an array of 97 layers of $1.6 \mathrm{~mm}$ thick FR4 boards, for which the experimentally determined permittivity around the $1.3 \mathrm{GHz}$ design frequency was $\varepsilon_{h}=4.3$ with loss tangent $\tan \delta=0.02$. The boards of FR4 have been printed with parallel metallic strips with width $w_{s}=0.83 \mathrm{~mm}$. The metallic strips are tilted by $\pm 45^{\circ}$ with respect to the interfaces, and thus the strips in adjacent FR4 boards are mutually orthogonal. The lattice constant is $a=3.2 \mathrm{~mm}$, and the height of the substrate is $L=2.6 a=8.32 \mathrm{~mm}$. As illustrated in Fig. 1(a), the metallic strips are supposed to be connected with good

${ }^{\text {a)} E l e c t r o n i c ~ m a i l: ~ m a r i o . s i l v e i r i n h a @ c o . i t . p t . ~}$
Ohmic contact to a metallic ground plane. In practice, such configuration may be difficult to reproduce using planar technology. To avoid this difficulty, we have mimicked the ground plane by printing an additional metallic strip running along the $x$-direction at the lower edge of each FR4 board. This ensures a good Ohmic contact between all the strips in the same board. In addition, to ensure Ohmic contact between the stacked FR4 boards, each FR4 board was perforated at 17 specific spots next to the lower edge (these spots can be identified in the lower panel of Fig. 1), and copper wires (running along the $y$-direction) were inserted through these spots and soldered to the boards. Finally, the bottom face of the fabricated prototype was coated with a silver paint. Since the spacing between the copper wires is very small as compared to the wavelength, such configuration effectively behaves as a continuous ground plane.

In order to characterize the properties of the material, we have investigated the propagation of transverse electric (TE)
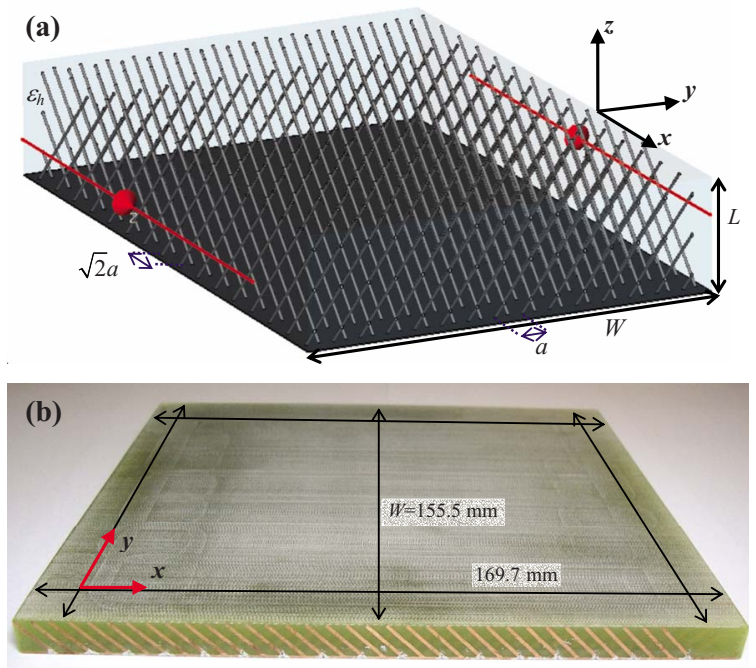

FIG. 1. (Color online) (a) A grounded metamaterial slab is placed in between two horizontal dipole antennas. The structured material is formed by an array of crossed metallic wires parallel to the xoz plane and are tilted by $\pm 45^{\circ}$ with respect to the interfaces. The wires are embedded in a host material with relative permittivity $\varepsilon_{h}$. (b) Photo of the fabricated prototype formed by 97 FR4 boards printed with metallic strips. 


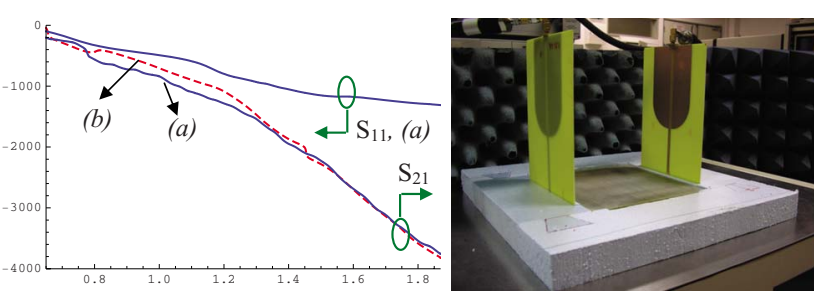

Phase [deg] vs. Frequency [GHz]

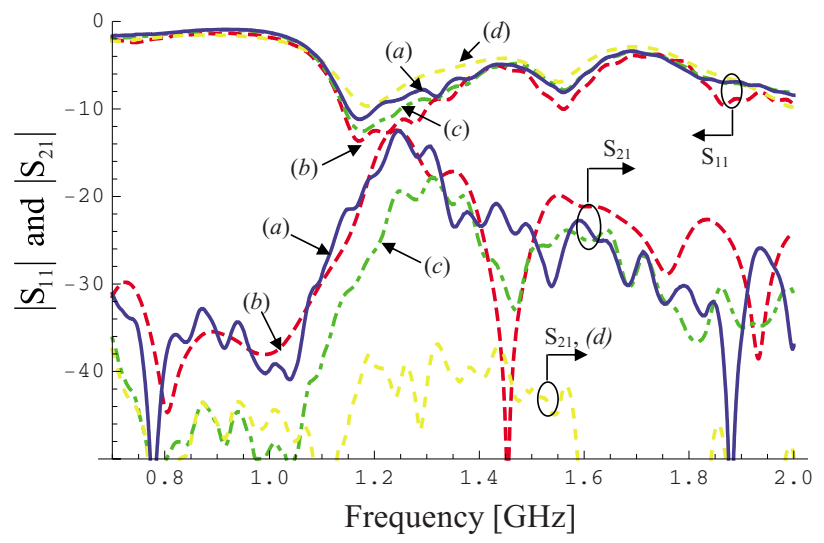

FIG. 2. (Color online) Lower panel: Amplitude of the $S$-parameters in dB. (a) Measured results. (b) CST MICROWAVE STUDIO full wave simulations. (c) Measured results when an absorber is placed in between the two antennas. (d) Same as (c) but the prototype is rotated by $90^{\circ}$. Top left panel: Phase for cases (a) and (b). Top right panel: photo of the experimental setup.

guided modes in the structured substrate. It is well known that a grounded dielectric slab with relative permittivity $\varepsilon$ only supports guided modes when $L>\lambda_{0} / 4 \sqrt{\varepsilon-1}$. Thus, a grounded substrate with the same permittivity as the FR4 boards only supports the TE-guided modes for frequencies larger than $4.98 \mathrm{GHz}$. As theoretically shown in Ref. 6, the presence of the crossed wire mesh may result in a dramatic increase of the effective permittivity and consequently in a significant reduction of the TE-mode cutoff frequency. In order to experimentally verify this property and measure the dispersion characteristic of the guided modes, the structured substrate was excited by a horizontal dipole antenna oriented along the $x$-direction and placed $5 \mathrm{~mm}$ away from the metamaterial substrate. The transmitted field was measured by an identical dipole placed at the other end of the substrate (top panel of Fig. 1). Actually, the antennas used in the experiment were not wire dipoles but instead were printed in FR4 boards as well. Each printed dipole is connected to the vector network analyzer (VNA) through a "balun" that makes the proper transition between the VNA coaxial cable and the coplanar printed line that feeds the dipole. In order that the "baluns" are not detuned by the presence of the structured substrate, it is convenient that the dielectric boards with the feeding circuit are oriented in such a way that they are normal to the $y$-direction (top right panel of Fig. 2). The free-space resonance frequency of the printed dipoles is approximately $1.2 \mathrm{GHz}$.

The phase and amplitude of the measured $S$-parameters are depicted in the top left and lower panels of Fig. 2, respectively [curves (a)]. The calibration planes are at the antenna SMA connectors. Note that $\left|S_{11}\right|$ is a measure of the fraction of power accepted by the input antenna, whereas $\left|S_{21}\right|$ is a measure of the coupling between the two dipoles. It is important to mention that even if there is an excellent coupling between the two dipoles, the level of $\left|S_{21}\right|$ does not need to be close to $0 \mathrm{~dB}$, since part of the power accepted by the input antenna is either radiated as space wave or dissipated in the dielectrics and metals, and only the remaining power is received by the test antenna, being the main propagation mechanism of guided wave. It is seen in Fig. 2 that for frequencies below $1.1 \mathrm{GHz}$ the level of $\left|S_{21}\right|$ is below $-30 \mathrm{~dB}$, showing that for these frequencies the coupling between the dipoles is very low. This is understandable since for low frequencies the TE-guided modes are cut off. However, around $1.25 \mathrm{GHz}$ there is a dramatic increase in the level of $\left|S_{21}\right|$ which approaches $-12 \mathrm{~dB}$, suggesting that the structure may support a guided mode above $1.25 \mathrm{GHz}$, notwithstanding the electrically small thickness of the substrate $\left(L=\lambda_{0} / 29\right)$. This property stems from the anomalously high effective permittivity of the structured material. ${ }^{6}$ For frequencies above $1.35 \mathrm{GHz}$, the $\left|S_{21}\right|$ level remains significant, even though it deteriorates with increasing frequency, in part, because the dipoles were tuned to $1.2 \mathrm{GHz}$, and thus they tend to radiate poorly for frequencies relatively far from 1.2 $\mathrm{GHz}$. We have experimentally verified with a different set of printed dipoles tuned to $1.5 \mathrm{GHz}$ that the onset of propagation of guided modes is still at $1.25 \mathrm{GHz}$, whereas the $\left|S_{21}\right|$ level may be improved around $1.5 \mathrm{GHz}$ (not shown here). Another reason for the deterioration of the $\left|S_{21}\right|$ level is that slightly above the cutoff frequency the guided modes become very confined inside the structured material, and thus it is difficult to launch the guided mode from the air region. This is supported by the extremely fast variation of the $S_{21}$ phase slightly above $1.25 \mathrm{GHz}$, suggesting that the guided mode has an extremely short wavelength and consequently is highly confined to the structured material because of the very large effective permittivity.

The reported experimental data concur very well with the results obtained with a full wave commercial electromagnetic simulator ${ }^{7}$ [curves (b) in Fig. 2]. In the numerical simulations, the ground plane was assumed continuous. The excellent agreement between the numerical and experimental results demonstrates that our implementation of the ground plane effectively mimics an ideal continuous ground plane.

In order to show that the main propagation mechanism between the two dipoles is indeed a guided wave, we have placed an $80 \mathrm{~mm}$ thick microwave absorber block in between the two antennas, $7 \mathrm{~mm}$ above the prototype. The purpose of the absorber was to block the space wave radiated by the dipole. The corresponding measured $S$-parameters are shown in Fig. 2 [curves (c)]. It is seen that except near $1.25 \mathrm{GHz}$, the $\left|S_{21}\right|$ level is little affected by the presence of the absorber, consistently with our expectations that the dominant propagation mechanism is the guided mode. The drop of the $\left|S_{21}\right|$ close to the cutoff is understandable, since at the cutoff frequency most of the energy of the guided mode propagates in the air region, and thus is also blocked by the absorber. To demonstrate in a conclusive manner that the enhancement of the $\left|S_{21}\right|$ level is due to the emergence of guided modes, we have rotated the prototype by $90^{\circ}$ so that the dipole antennas become orthogonal to the FR4 boards with the printed strips. In this case, it is expected that the interaction of the metallic strips with the dipoles is very weak, and thus the prototype should behave in the same manner as a homogeneous grounded substrate with the same permittivity as the FR4 boards. The measured results corresponding to this scenario 


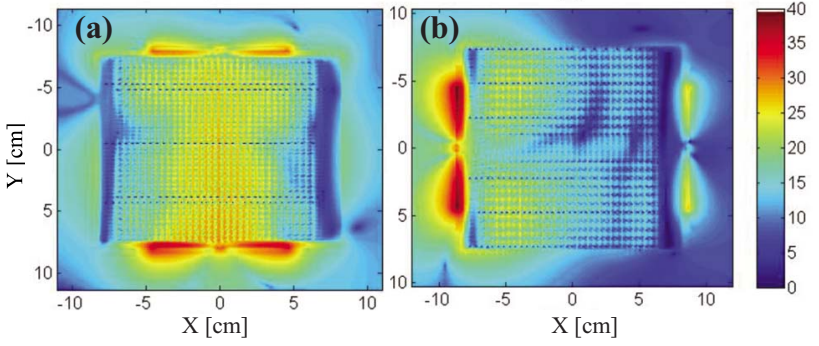

FIG. 3. (Color online) CST MICROWAVE STUDIO simulations at $1.25 \mathrm{GHz}$ for the total electric field amplitude at the midheight of the artificial substrate for dipole antennas (a) parallel to the $x$-axis and (b) parallel to the $y$-axis.

are depicted in the lower panel of Fig. 2 [curves (d)], and demonstrate that indeed the $\left|S_{21}\right|$ level is close to $-40 \mathrm{~dB}$, consistent with the fact that a homogeneous dielectric substrate with $\varepsilon_{h}=4.3$ only supports the TE-guided modes above $4.98 \mathrm{GHz}$. The results obtained with CST MICROWAVE STUDIO completely confirm these findings. In Fig. 3, we plot the total electric field amplitude at $1.25 \mathrm{GHz}$ along the midheight cut of the structured substrate for the cases in which the dipoles are parallel to either the $x$-axis or to the $y$-axis. In these simulations, the region in between the printed dipoles was not blocked by the microwave absorber. The plots demonstrate how in the case in which the dipoles are parallel to the FR4 boards [panel (a)], the wave is able to penetrate into the structured substrate permitting a good coupling between the two dipoles. This situation contrasts markedly with the case in which the dipoles are perpendicular to the FR4 boards [panel (b)], for which the guided mode cannot be launched and the wave cannot propagate along the substrate.

It is possible to calculate the dispersion characteristic of the guided modes from the experimental data. A straightforward analysis shows that the propagation constant of the guided mode, $k_{y}$, is such that $k_{y} \approx 1 / W[2 \arg (1-\rho)-\arg (\tau)$ $+C]$, where $W$ is the width of the substrate (see Fig. 1), $C$ is a constant chosen so that $k_{y}=\omega / c$ at the cutoff frequency of the guided mode, and $\rho$ and $\tau$ are the $S_{11}$ and $S_{21}$ parameters referred to the input ports of the printed dipoles, respectively (these parameters are obtained by proper de-embedding of the experimental data obtained at the antenna SMA connectors $^{8}$ ). The extracted dispersion characteristic of the guided mode is depicted in Fig. 4 (blue curve). It was assumed in the extraction procedure that the cutoff frequency is $1.25 \mathrm{GHz}$, consistent with the results of Fig. 2. In Fig. 4, we have also plotted the dispersion characteristic calculated with CST MICROWAVE STUDIO, ${ }^{7}$ obtained by analyzing a single cell of the periodic substrate (red curve), as well as the dispersion characteristic obtained using the analytical model described in our previous work ${ }^{6,9}$ (black curve). It is seen that the results obtained with the different methods concur very well, and that the value of $k_{y} c / \omega$ may be significantly larger than unity for frequencies slightly above the cutoff

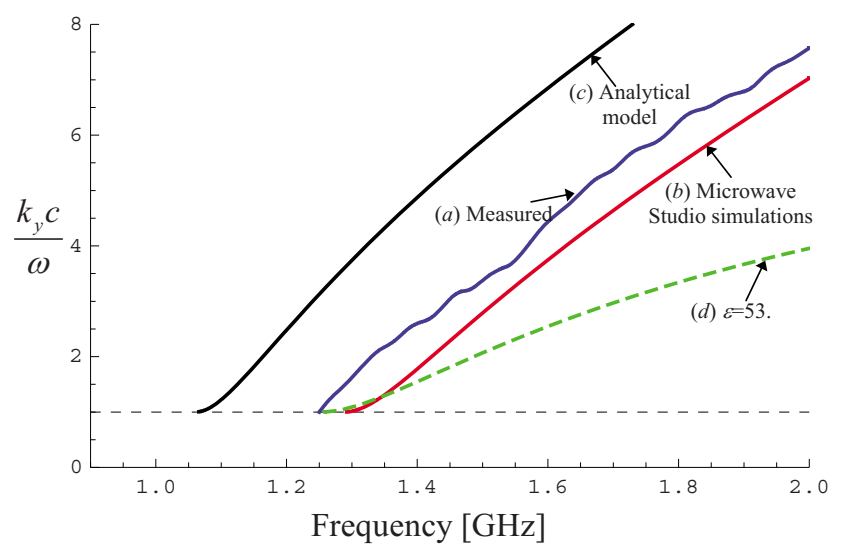

FIG. 4. (Color online) Dispersion characteristic of the guided mode supported by the structured material. (a) Measured result. (b) Full wave simulation with Ref. 7. (c) Theoretical result. (d) Dispersion for a dielectric slab with $\varepsilon=53$.

frequency. This supports the fact the guided mode becomes highly confined in the structured material. In curve (d) of Fig. 4, we show the dispersion characteristic of a grounded dielectric slab $(\mu=1)$, with the same cutoff frequency as the prototype. The permittivity of the equivalent dielectric slab is $\varepsilon=53.0$, which demonstrates how the crossed wire mesh may, in fact, enhance in a dramatic manner the electric properties of the host medium. As discussed in Ref. 6, much larger values of the permittivity may be easily obtained by increasing the density of wires. It is interesting to note that the slope of $k_{y} c / \omega$ versus frequency is much larger for the structured material than for the equivalent dielectric slab. This property is a consequence of the fact that the response of the bulk structured material is anisotropic, even for propagation in the yoz plane. Quite remarkably, this property enables a greater confinement of the electromagnetic fields inside the metamaterial slab and thus suggests interesting applications of the proposed structure as an ultrasubwavelength waveguide.

This work is supported in part by Fundação para a Ciência e a Tecnologia, Grant No. POSC/EEACPS/61887/2004. The authors gratefully acknowledge technical support from Carlos Brito, Vasco Fred, and António Almeida.

${ }^{1}$ J. Pendry, Phys. Rev. Lett. 85, 3966 (2000).

${ }^{2}$ M. Silveirinha and N. Engheta, Phys. Rev. Lett. 97, 157403 (2006).

${ }^{3}$ P. A. Belov, Y. Hao, and S. Sudhakaran, Phys. Rev. B 73, 033108 (2006).

${ }^{4}$ C. Rockstuhl, F. Lederer, C. Etrich, T. Pertsch, and T. Scharf, Phys. Rev. Lett. 99, 017401 (2007).

${ }^{5}$ J. B. Pendry, D. Schurig, and D. R. Smith, Science 312, 1780 (2006).

${ }^{6}$ M. G. Silveirinha and C. A. Fernandes, Phys. Rev. B 78, 033108 (2008).

${ }^{7}$ CST MICROWAVE STUDIO 2006, CST GmbH, http://www.cst.com.

${ }^{8}$ D. M. Pozar, Microwave Engineering (Wiley, New York, 2006).

${ }^{9}$ The model proposed in Ref. 6 assumes that the inclusions are wires and not strips; we have taken the radius of the wires equal to $R=\pi w_{s}$, so that the perimeter of the cross section of the inclusions is the same. 